\title{
Value of UDT
}

The UDT should be performed to find answers for the following questions:

- How is the urodynamic situation, cystometric capacity, detrusor pressure development?

- Is the emptying technique used appropriate and safe?

- Why does incontinence, recurrent infection, trabeculation/diverticula formation, autonomic dysreflexia develop?

- If video-urodynamics is used, is vesicoureteral reflux or other pathology in the LUT found? Is the bladder neck closed during filling and open during voiding? 